Historic, Archive Document

Do not assume content reflects current scientific knowledge, policies, or practices. 



\section{SLATE'S FIELD SEED \\ Price List}

TERMS...-Subject to being unsold, market changes, and our usual non-warranty, we offer the following seeds. Every lot of field seeds of ten pounds or more is tagged in accordance with the Agricultural Seed Laws of Virginia and North Carolina. Samples with Purity and Germination tests will be sent upon request. Prices on Field Seeds fluctuate. This list is issued every week during the season and we shall be glad to send a revised copy to anyone upon request.
SHIPPING CHARGES.-...-When quotations have "Post paid" above them, it means that we will deliver goods at these prices by parcel post without any extra charge of any kind. Freight or Express above a quotation means that customer is to pay delivery charges but no charge for sacks. Those who wish may order by these prices and add the exact amount of postage necessary. kind

SACKS FREE... We make no extra charge for sacks of any
January 15, 1925

\section{Clovers}

Alfalfa

Alsike

Burr

Japan

Red, American

Sapling

White Dutch.

White Sweet.

\section{Seed Corn}

Boone County.

Va. White Dent

Hickory King.

Imp. Golden Dent..

Blounts Prolific.

Biggs Seven Ear

Early White Dent

Ensilage

\section{Peanuts}

Virginia.

Valencia

Spanish

\section{Seed Oats}

Fulghum

Appler

Red Rust Proof

Burt or 90 Day.

White Spring

\section{Soup Beans}

White Navy.

White Kidney

Red Kidney

\section{Edible Cow Peas}

Large Blackeyes

Sugar Crowder

\section{Cotton}

Cleveland Big Boll.

\section{Sorg̉hum}

Early Amber

Sugar Drip.

Prices Change

Write for a new list at any time

\begin{tabular}{ccr}
\multicolumn{2}{c}{ Postpaid } & $\begin{array}{r}\text { Exp. } \\
\text { Lb. }\end{array}$ \\
.40 & 3.10 & 15.00 \\
.40 & 3.10 & 16.50 \\
.20 & 1.75 & 1.60 \\
.40 & 325 & 7.00 \\
.50 & 4.50 & 23.50 \\
.50 & 4.55 & 24.50 \\
.65 & 6.00 & 36.00 \\
.30 & 2.80 & 10.40
\end{tabular}

Postpaid

Gal. Pk.

$.75 \quad 1.25$

$.75 \quad 1.25$

$.80 \quad 1.35$

$.75 \quad 1.25$

$.75 \quad 1.25$

$80 \quad 135$

$.85 \quad 1.40$

$.75 \quad 1.25$

Postpaid By Exp.

Lb. 5 lbs. $100 \mathrm{lbs}$.

$\begin{array}{lll}.30 & 1.00 & 15.00\end{array}$

$\begin{array}{lll}.30 & .90 & 13.50\end{array}$

$\begin{array}{lll}.25 & .85 & 12.00\end{array}$

By Frt. or Exp.

Bu. $\quad 10 \mathrm{Bu}$.

$1.30 \quad 1.25$

$1.20 \quad 1.25$

$1.05 \quad 1.00$

$1.15 \quad 1.10$

$.95 \quad .90$

Postpaid By Exp

Lb. 5 lbs. 100 lbs.

$\begin{array}{lll}25 & 1.00 & 9.00\end{array}$

$\begin{array}{lll}.25 & 1.10 & 10.00\end{array}$

$\begin{array}{lll}.25 & 1.10 & 10,00\end{array}$

Postp'd by exp.

Lb. $10 \mathrm{lbs}$. $109 \mathrm{lbs}$.

$.25 \quad 1.00$

$.25 \quad 1.00$

By Frt. or Exp. Bu. $10 \mathrm{Bu}$. $2.00 \quad 1.90$

Postp'd by exp. Lb. $10 \mathrm{lbs}$. $100 \mathrm{lbs}$. $\begin{array}{lll}.20 & 1.25 & 6.00\end{array}$ $\begin{array}{lll}.25 & 2.00 & 10.00\end{array}$

\section{Grasses}

Herds Grass.

Italian Rye.

Perennial Rye.

Meadow Fescue.

Timothy

Orchard

Kentucky Blue

Tall Meadow Oat

Carpet Grass

Sudan Grass

Bermuda Grass

Creeping Bent

Fine Leaved Fescue

\section{Mixtures}

Mixture No. 1 Pasture........

Mixture No. 2 Hay.

Mixture No. 3 Pasture........

Mixture No. 4 Hay.

Mixture No. 5 Hog Pasture

Mixture No. 6 Hog Pasture

\section{Vetch}

Hairy or Winter.

\section{Millet}

Golden or German..

Pearl or Cattail

\section{Miscellaneous Seeds}

\section{Dwarf Essex Rape.}

Japanese Buckwheat

White Kaffir Corn..

Evergreen Broom Corn..

Teosinte

Sunflower

\section{Field Beans}

Mam. Yellow Soys'.

Tarheel Black

Miam. Brown.

Laredos Soys.

Mung Beans.

Early Speckled Velvet

\begin{tabular}{|c|c|c|}
\hline & \multirow{2}{*}{$\begin{array}{r}\text { Exp. } \\
100 \mathrm{lbs} . \\
16.00\end{array}$} \\
\hline \multicolumn{2}{|c|}{$\begin{array}{l}\text { Postpaid } \\
\text { Lb. } 10 \mathrm{lbs} .\end{array}$} & \\
\hline .25 & 2.00 & 15.00 \\
\hline .25 & 2.00 & 15.00 \\
\hline .30 & 2.50 & 15.00 \\
\hline .25 & 1.50 & 10.00 \\
\hline .30 & 2.60 & 21.00 \\
\hline .50 & 4.00 & 35.00 \\
\hline .35 & 3.00 & 18.00 \\
\hline .45 & 4.00 & 35.00 \\
\hline .25 & 1.50 & 9.00 \\
\hline .60 & 5.00 & 45.00 \\
\hline .75 & 7.00 & 60.00 \\
\hline .60 & 5.50 & 45.00 \\
\hline \multicolumn{2}{|c|}{ Postpaid } & Exp, \\
\hline .30 & 3.25 & 26.00 \\
\hline .30 & 2.85 & 22.30 \\
\hline .35 & 3.25 & 26.00 \\
\hline .30 & 2.85 & -22.50 \\
\hline .15 & 1.25 & 7.50 \\
\hline .15 & 1.15 & 6.00 \\
\hline \multicolumn{2}{|c|}{ Postpaid } & $\begin{array}{l}\mathrm{E} \mathrm{p}_{\text {ibs }} \\
100 \mathrm{lbs}\end{array}$ \\
\hline .25 & 1.75 & 13.00 \\
\hline
\end{tabular}

Postp'd by exp

Lb. $10 \mathrm{lbs} .100 \mathrm{lbs}$.

$\begin{array}{lll}.20 & 1.25 & 7.00\end{array}$

Postp'd by exp.

Lb. $10 \mathrm{lbs}$. $100 \mathrm{lbs}$.

$\begin{array}{lll}.25 & 1.60 & 12.00\end{array}$

$\begin{array}{lll}.20 & .90 & 2.50\end{array}$

$\begin{array}{lll}.20 & .90 & 10.00\end{array}$

$\begin{array}{lll}.25 & 1.00 \quad 15.00\end{array}$

$\begin{array}{lll}.60 & 2.50 \quad 45.00\end{array}$

$\begin{array}{lll}.20 & .85 & 10.60\end{array}$

ByFrt. or Exp

Bu. $10 \mathrm{Bu}$.

\section{PRICES}

LATER 
SLATE'S FIELD SEED LIST

\section{Cow Peas}

Whippoorwills

Mixed Cow Peas

Peas \& Soy Beans Mixed

Cánada Field

\section{Seed Irish Potatoes}

Irish Cobbler

Early Rose

Red Bliss

\begin{tabular}{|c|c|c|c|c|}
\hline Oni & Sets & & \multicolumn{2}{|c|}{$\begin{array}{l}\text { Postpaid } \\
\text { Gal. }\end{array}$} \\
\hline Whi & tom. & & .80 & 1.2 \\
\hline & “. & .25 & .75 & 1.1 \\
\hline & “ & .25 & .75 & 1.1 \\
\hline
\end{tabular}

\section{Inoculation}

1 Acre Farm-O-germ

3 Acre Farm-C-rerm
$\underset{\mathrm{Bu}}{\mathrm{By} \text { Frt. or Exp. }}{ }_{10 \mathrm{Bu}}$

\section{PRICES \\ LATER \\ 4.25}

By Frt. or Exp

$\begin{array}{rrr}\mathrm{Pk} & \text { Bu. } \quad \text { Sk } \\ 75 & 170 & 3.65\end{array}$

$\begin{array}{lll}.75 & 1.70 & 3.65\end{array}$

$\begin{array}{lll}.75 & 1.70 & 3.65\end{array}$

\section{THE SLATE SE
SOUTH BOSTON,}

4.20

\section{Lawn Grass}

Perfection

Utility .

Special Shade.

Sandy Soil.

Cemetary Mixture

Conkeys Poultry Feeds

Buttermilk Starting Feeds $5 \mathrm{~s}$

Buttermilk Starting Feeds 10s

Buttermilk Starting Feeds 25s

Buttermilk Starting Feeds 100s

Chick Grains 100 lbs.

Buttermilk Growing Mash 100s

Growing Grains 100s

Buttermilk Laying Mash 100s

Meat Scrap 100 lbs.

Postpaid

1.00

2.50
Prepared Charcoal 50 lbs.

Oyster Shell 100 lbs.

Scratch Grains 100s

$\begin{array}{ccc}\text { Lb } & \begin{array}{c}\text { Postpaid } \\ 10 \mathrm{lbs}\end{array} & \begin{array}{c}\text { Exp. } \\ 100 \mathrm{lbs}\end{array} \\ .60 & 5.00 & 40.00 \\ .10 & 3.00 & 25.00 \\ .55 & 4.50 & 35.00 \\ .55 & 4.50 & 32.50 \\ .55 & 4.50 & 33.00\end{array}$

By Exp. Sack

1.10

2.00

5.25

4.85

4.75

4.75

4.50

5.00

2.25

1.25

4.25 